\title{
Treatment of class II division I patients using intermaxillary elastics
}

\author{
Konstantin Georgiev, Silviya Krasteva \\ Department of Orthodontics, Faculty of Dental Medicine, \\ Medical University Plovdiv, Bulgaria;
}

\section{Abstract}

Objective The objective of this study is to assess the cephalometric outcome of the application of intermaxillary elastics in class II division I patients.

Materials and methods The investigation comprised a sample of 16 patients with class II division 1 malocclusion treated nonextraction with fixed technique and intermaxillary elastics. Lateral cephalograms were taken before applying class II intermaxillary elastics and after reaching class I molar relationship We analyzed angular measurements for the anteroposterior position of the jaws (SNA, SNB, ANB angles) and the inclination of the occlusal plane in relation to the sella-nasion(SN) plane.

Results The Statistical Package for the Social Sciences (SPSS), Version 25 (2017) and Minitab Version 18.1 (2017) were used to analyze the data. The extent of the change in the SNA, ANB, SNB, and OcP/SN values before and after the treatment was examined through paired-samples t-tests. The results showed a significant reduction in the mean SNA angle $\left(79.56^{\circ} \pm 3.94\right.$ before vs. $78.43^{\circ} \pm 3.89$ after, $\left.p=0.012\right)$ and in the mean ANB angle ( $4.31^{\circ} \pm 1.87$ before vs. $3.37^{\circ} \pm 1.23$ after, $p=0.009$ ). A significant increase was observed in the OcP/SN angle $\left(14.25^{\circ} \pm 4.46\right.$ before vs. $16.0^{\circ} \pm 3.86$ after, $\left.p=0.008\right)$. The increase in the SNB angle was not significant $\left(76.22^{\circ} \pm 3.55\right.$ before vs. $76.32^{\circ} \pm 3.59$ after, $\left.p=0.068\right)$.

Conclusion The orthodontic treatment was found effective in reducing the SNA and ANB angles and in increasing the OcP/SN angle.

Keywords: Class II Division I, intermaxillary elastics, lateral cephalogram, occlusal plane 


\section{Introduction}

Many patients who seek orthodontic treatment exhibit a class II tendency (1). One of the most common clinical modalities for the correction of distal bite in patients treated with fixed appliances is the application of class II intermaxillary elastics (2). They can be classified as an additional active element which is attached to the frontal maxilla and posterior mandible aiming mainly to correct the anteroposterior discrepancy $(3,4,5)$. Along with their sagittal effect, they also produce a transversal and vertical movement of the teeth and arches $(6,7)$. The vertical component of the generated force causes extrusion of the lower molars and palatal inclination and extrusion of the upper incisors. These dental movements are the reason for the backwards rotation of the occlusal plane which is a side effect of the intermaxillary traction(8). In cases of prolonged usage labial inclination of the lower incisors can also be observed.

Depending on the individual patient's growth pattern, archwire selection and treatment needs there exist specific ways to attach them as well as types of elastics according to their size and force (3). A lot of studies $(6,9,10,11,12,13)$ claim that the effect of intermaxillary traction is mainly dentoalveolar especially in patients whose growth is almost finished or entirely concluded. The changes in the sagittal skeletal relationships and profile are not satisfactory. Despite their widespread usage we have found very few studies in the literature which focus mainly on their effects and the ones that we found were in combination with the Begg appliance $(9,14)$.

\section{Materials and methods}

The investigation comprised a sample of 16 patients (10 women and 6 men) aged between 14 and 19 years (mean age 16,62 years \pm 1.53 ) with class II division 1 malocclusion. All of them exhibited a skeletal distal bite due to an underdeveloped mandible. They underwent a standard nonextraction treatment with fixed technique(MBT 0,022-in slot prescription) and intermaxillary elastics. The usage of intermaxillary traction was introduced on $0.016 \times 0.022$ " upper and lower stainless steel archwires. The elastics used in all patients were $1 / 4$ " size exerting heavy force and attached between upper canine and lower first molar with a 24-hour recommended wear-time. To assess the cephalometric changes we made lateral cephalograms before applying class II intermaxillary elastics and after reaching class I molar relationship. We used SNA, SNB and ANB angles in order to evaluate the anteroposterior effect. To assess the change in the inclination of the occlusal plane we used the angle between the occlusal plane and Sella-Nasion (Ocp/Sn). We constructed the occlusal plane in accordance with Ricketts method - through the midpoint of the overlapping of the mesial cusps of the first molars and the first premolars(15).

\section{Aim}

The objective of this study is to assess the cephalometric outcome of the application of intermaxillary elastics in class II division I patients. 


\section{Results}

The treatment goals were achieved in all patients included in the current study. The Statistical Package for the Social Sciences (SPSS), Version 25 (2017) and Minitab Version 18.1 (2017) were used to analyze the data. The assumption of normality was observed in all variables (Kolmogorov-Smirnov $p$-values $<0.05$ ). The extent of the change in the SNA, ANB, SNB, and OcP/SN values before and after the treatment was examined through paired-samples t-tests. Statistical significance was accepted at $p<0.05$.

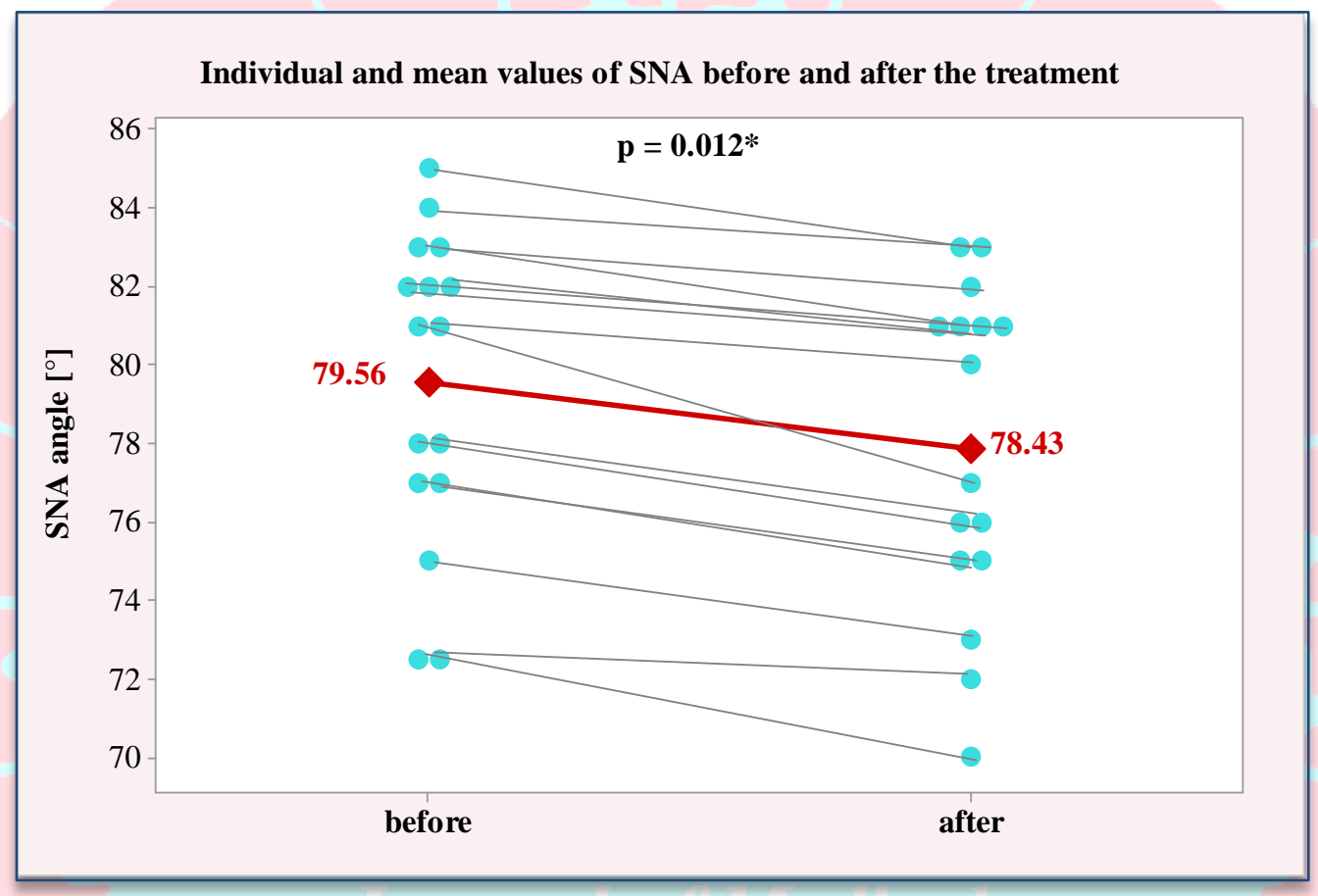

Figure 1. Values of SNA berofe and after the treatment

The results showed a significant reduction in the mean SNA angle $\left(79.56^{\circ} \pm 3.94\right.$ before vs. $78.43^{\circ} \pm 3.89$ after, $p=0.012)$ (Fig. 1) and in the mean ANB angle ( $4.31^{\circ} \pm 1.87$ before vs. $3.37^{\circ} \pm 1.23$ after, $\left.p=0.009\right)$ (Fig. 2). A significant increase (Fig. 3) was observed in the OcP/SN angle $\left(14.25^{\circ} \pm 4.46\right.$ before vs. $16.0^{\circ} \pm 3.86$ after, $\left.p=0.008\right)$. The increase in the SNB angle was not significant $\left(76.22^{\circ} \pm 3.55\right.$ before vs. $76.32^{\circ} \pm 3.59$ after, $\left.p=0.068\right)$. 


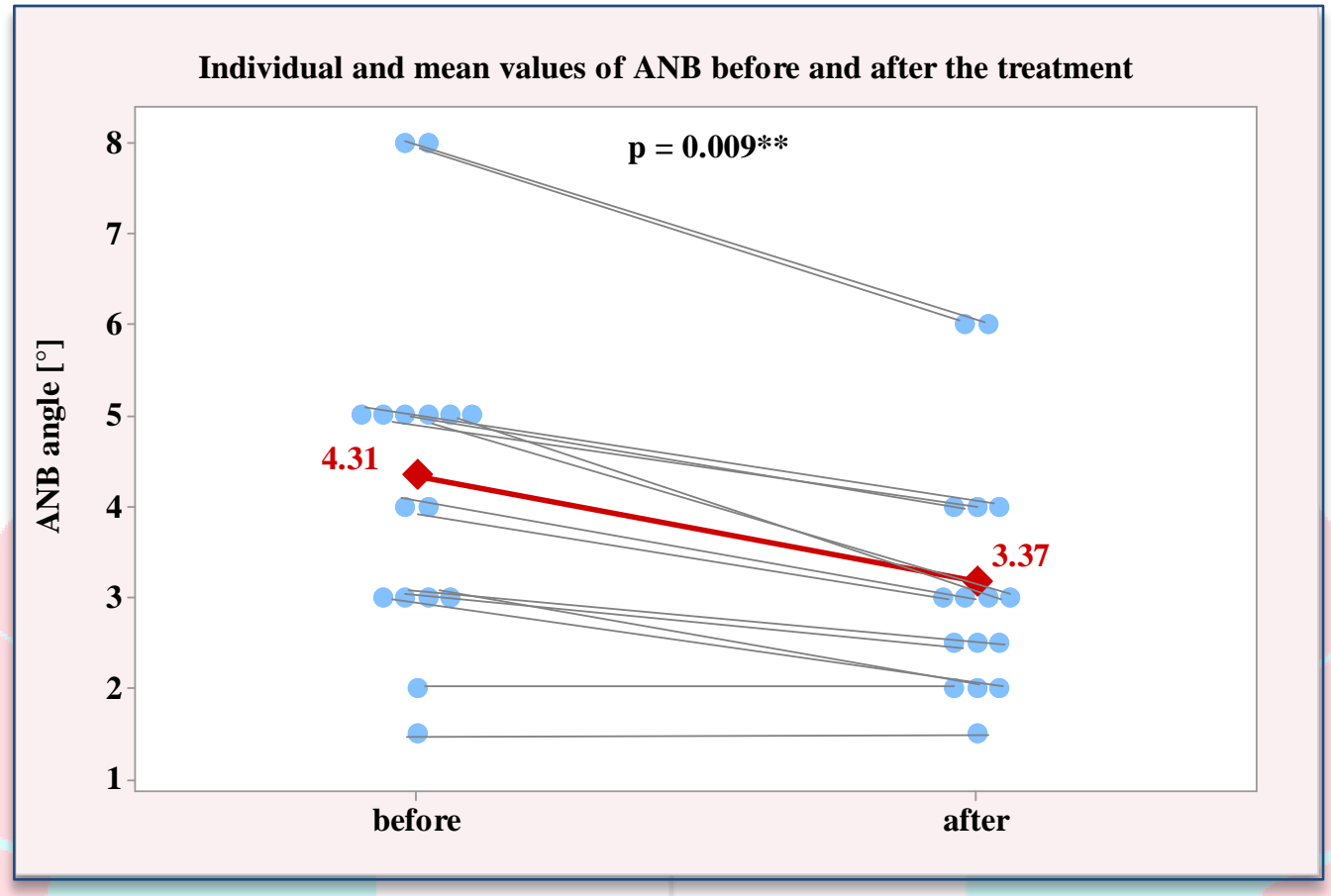

Figure 2. Values of ANB berofe and after the treatment

Individual and mean values of $\mathrm{OcP} / \mathrm{SN}$ before and after the treatment

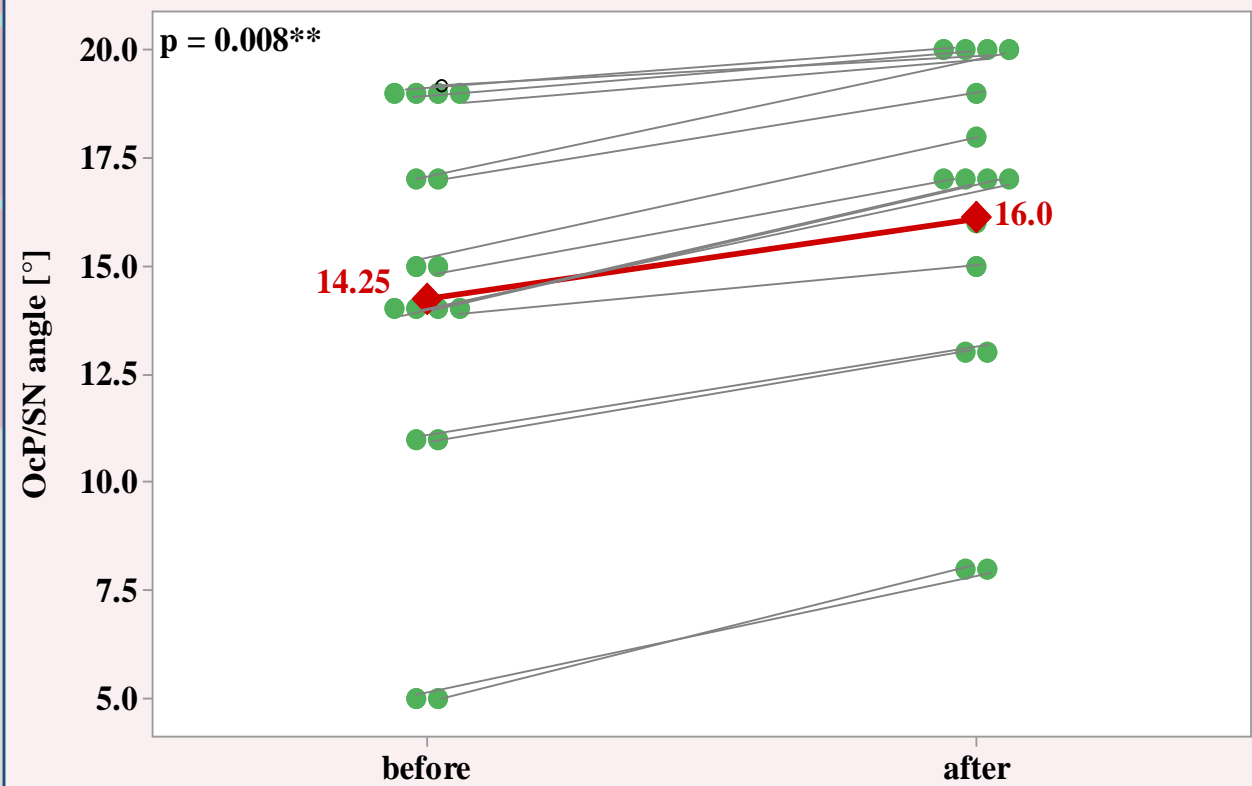

Figure 3. Values of OcPISN berofe and after the treatment 


\section{Discussion}

Our results correspond with these from other similar studies. The $0.97^{\circ}$ decrease of ANB angle correlates with the $1,62^{\circ}$ decrease reported by Reddy (9). The normalization of this mandibulomaxillary discrepancy cannot be attributed to an increase in SNB angle but to a decrease in the SNA angle as is proved by most of the studies in the literature as well $(9,12,14,16)$. This finding suggests that the class II elastics lead to a restraint in the upper jaw and not so much mandibular advancement. Only Ozbilek reports a stimulating effect on the lower jaw but he uses skeletally anchored and not dentally anchored elastics (4). Our decrease in SNA angle $\left(1,13^{\circ}\right)$ is bigger than the ones in other studies (9) but since the position of point A is dependent on the inclination of the upper incisors, it is unlikely that this result indicates an orthopedic effect.

Backward occlusal plane rotation is reported in all other studies on the effect of class II elastics application. The clockwise rotation of the occlusal plane plays a role in the vertical dimension change. This is the reason why the utilization of class II elastics should consider the gingival display during physiological rest position and smiling. These results confirm that class II elastics usage should take into account the individual morphological and esthetic needs of each patient.

\section{Conclusion}

The orthodontic treatment was found effective in reducing the SNA and ANB angles and in increasing the OcP/SN angle.

Conflict of interest Authors declares that they have no conflict of interest.

Funding The work was supported by the Medical University of Plovdiv, Bulgaria.

\section{References}

1. Petrunov V. Distal bite in children aged between 7 and 14 years old - incidence and clinical characteristics. Contemporary Dentistry. 2013; 1(26):33.

2. Combrink FJ, Harris AM, Steyn CL, Hudson AP. Dentoskeletal and soft-tissue changes in growing class II malocclusion patients during nonextraction orthodontic treatment. SADJ 2006; 61(8):344350.

3. Uzel A, Uzel I, Toroglu MS. Two different applications of Class II elastics with nonextraction segmental techniques. Angle Orthod 2007; 77(4):694-700.

4. Ozbilek S, Gungor A, Celik S. Effects of skeletally anchored Class II elastics: A pilot study and new approach for treating Class II malocclusion. Angle Orthod 2017; 87(4):505-512.

5. Langlade M. Optimisation of orthodontic elastics. Publisher:New York:GAC International 2000.

6. Nelson B, Hansen K, Hägg U. Class II correction in patients treated with Class II elastics and with fixed functional appliances: A comparative study. Am J Orthod Dentofacial Orthop 2000; 118(2):142-149.

7. Ellen E, Schneider B, Selike T. A comparative study of anchorage in bioprogressive versus standard edgewise treatment in Class II correction with intermaxillary elastic force. Am J Orthod Dentofacial Orthop 1988; 114(4):430-436. 
8. Aras I, Pasaoglu A. Class II subdivision treatment with the Forsus Fatigue Resistant Device vs intermaxillary elastics. Angle Orthod 2017; 87(3):371-376.

9. Reddy $\mathrm{P}$, Kharbanda OP, Duggal R, Parkash H. Skeletal and dental changes with nonextraction Begg mechanotherapy in patients with Class II Division 1 malocclusion. Am J Orthod Dentofacial Orthop 2000; 118(6):641-648.

10. Bratu C, Fleser C, Glavan F. The effect of intermaxillary elastics in orthodontic therapy. TMJ 2004; 54(4):406-409.

11. Janson G, Sather R, Fernandes T, Branco N, Freitas M. Correction of Class II malocclusion with Class II elastics: A systematic review. Am J Orthod Dentofacial Orthop 2013; 143(3):383-92.

12. Nelson B, Hansen K, Hagg U. Overjet reduction and molar correction in fixed appliance treatment of Class II, Division 1, malocclusions: sagittal and vertical components. Am J Orthod Dentofacial Orthop 1999; 115(1):13-23.

13. Serbesis-Tsarudis C, Pancherz H "Effective" TMJ and Chin Position Changes in Class II Treatment Orthodontics versus Orthopedics. Angle Orthod 2008; 78(5):813-818.

14. Meistrell ME Jr, Cangialosi TJ, Lopez JE, Cabral-Angeles A. A cephalometric appraisal of nonextraction Begg treatment of Class II malocclusions. Am J Orthod Dentofacial Orthop 1986; 90(4):286-95.

15. Jacobson A. Radiographic cephalometry from basics to 3-D imaging. 2nd edition. Publisher: Canada:Quintessence Publishing; 2006.

16. Jayachandran S, Wiltshire WA, Hayasaki SM, Pinheiro FH. Comparison of AdvanSync and intermaxillary elastics in the correction of Class II malocclusions: A retrospective clinical study. Am J Orthod Dentofacial Orthop 2016; 150(6):979-988.

\section{Corresponding author:}

Dr Konstantin Georgiev,

Faculty of Dental medicine-Plovdiv, Blvd Hristo Botev 3

e-mail: konstantinvanev@gmail.com 